\title{
MONOTONICITY OF SOLUTIONS OF VOLTERRA INTEGRAL EQUATIONS IN BANACH SPACE $\left(^{(}\right)$
}

\author{
BY \\ AVNER FRIEDMAN
}

1. Introduction. We shall consider Volterra integral equations

$$
x(t)=x_{0}-\int_{0}^{t} h(t-\tau) A x(\tau) d \tau
$$

where $x_{0}$ and $x(t)$ belong to a complex Banach space $X, h(t)$ is a complex-valued function and $A$ is an operator in $X$, generally unbounded. We denote by $B(X)$ the Banach space of bounded linear operators in $X$, and by $I$ the identity operator in $X$. An operator-valued function $S(t)$, which belongs to $L^{1}(0, b ; B(X))$ for any $b>0$, is called a fundamental solution of (1.1) if

$$
S(t)=I-A \int_{0}^{t} h(t-\tau) S(\tau) d \tau
$$

for almost all $t$. In this definition it is assumed, of course, that the integral on the right-hand side of (1.2) is in the domain of $A$.

In a recent paper [6], Friedman and Shinbrot have studied the equation (1.1) even in the more general case where $x_{0}$ and $A$ depend on $t$ and $\tau$, respectively. They proved theorems of existence, uniqueness, differentiability and asymptotic behavior of solutions. They also constructed fundamental solutions and derived asymptotic bounds for them. We recall [6] that if $x_{0}$ is in the domain of $A^{\mu}$, for some $\mu>0$, then the solution of (1.1) is given by $S(t) x_{0}$.

The purpose of the present paper is to derive monotonicity theorems for solutions of (1.1). We shall generalize some of the monotonicity theorems of Friedman [2] (see also [4]) from the case $X=R^{1}$ ( $R^{1}$ the one-dimensional Euclidean space) to the case where $X$ is any Banach space.

In $\$ 2$ we give some auxiliary results. These results are concerned with Volterra equations in one-dimension (i.e., $X=R^{1}$ ). In particular, we study the behavior of the solutions with respect to a certain parameter.

In $\S 3$ we give an integral formula for $S(t)$ in case $A$ is a bounded operator. For $A$ unbounded, we construct a fundamental solution as a limit of fundamental solutions corresponding to the bounded operators $A(I+A / n)^{-1}$. We prove that the fundamental solution coincides with the fundamental solution of [6, Chapter 1]

Received by the editors April 9, 1968.

(1) This work was partially supported by the National Science Foundation NSF GP-5558. 
or of [6, Chapter 2] provided the assumptions of [6, Chapter 1] or of [6, Chapter 2], respectively, are satisfied.

In $\S 4$ we derive a formula for $S(t)$ in case $A$ is selfadjoint. As a by-product, we obtain monotonicity theorems for $\left(S(t) x_{0}, x_{0}\right)$.

In $\$ 5$ we drop the assumption that $A$ is selfadjoint. Instead we assume that the resolvent $(\lambda I-A)^{-1}$ exists for all $\lambda$ except for a sequence $\left\{\mu_{k}\right\}$ of poles, and $0<\mu_{1}$ $<\mu_{2}<\cdots, \mu_{k} \rightarrow \infty$ as $k \rightarrow \infty$. We obtain a formula for the solution $T(t) x_{0}$ of (1.1) and then derive monotonicity theorems for $f_{0}\left(T(t) x_{0}\right)$; here $f_{0}$ is a bounded linear functional in $X$.

In $\S 6$ we give some additional results obtainable with the methods of the previous sections, and some applications to control theory.

2. Auxiliary lemmas. A real-valued function $f(t)$ is said to be completely monotonic in an interval $[a, b)$ if $f \in C^{\infty}[a, b)$ and for all $n \geqq 0$,

$$
(-1)^{n} d^{n} f(t) / d t^{n} \geqq 0 \quad \text { for all } t \in[a, b) .
$$

Similarly one defines complete monotonicity in intervals $(a, b),[a, b]$. We recall the following results (see [9]):

LEMMA 2.1. If $f(t)$ is completely monotonic in an interval $(a, b)$ then $f(t)$ is analytic in $(a, b)$.

LEMMA 2.2. A function $f(t)$ is completely monotonic in the interval $[0, \infty)$ if and only if

$$
f(t)=\int_{0}^{\infty} e^{-\lambda t} d \phi(\lambda)
$$

where $\phi(\lambda)$ is a bounded nondecreasing function.

LEMMA 2.3. If $\left\{f_{m}(t)\right\}$ is a sequence of completely monotonic functions in $(a, b)$ and if $f(t)$ is a continuous function in $(a, b)$ such that, for each $t \in(a, b), f_{m}(t) \rightarrow f(t)$ as $m \rightarrow \infty$, then $f(t)$ is completely monotonic in $(a, b)$.

Setting $\Delta_{\eta}^{1} f(t)=f(t+\eta)-f(t)$,

$$
\Delta_{\eta}^{m+1} f(t)=\Delta_{\eta}^{m}\left(\Delta_{\eta} f(t)\right),
$$

the assertion of the last lemma is a consequence of the fact (see [9]) that $f(t)$ is completely monotonic if, for any integer $m \geqq 1$ and $\eta>0$,

$$
(-1)^{m} \Delta_{\eta}^{m} f(t) \geqq 0 \text { for } a<t<b-m \eta \text {. }
$$

In view of Lemma 2.1 , if $f(t)$ is a completely monotonic function in $(a, b)$ which does not vanish identically, then $f(t)>0$ for all $t \in(a, b)$.

We shall need the following result of Miller [8]:

LEMMA 2.4. If $f(t)$ is a nonzero completely monotonic function in $[0, \infty)$, then $\log f(t)$ is a convex function. 
Proof. We have to show that

$$
g(t) \equiv f(t) f^{\prime \prime}(t)-\left(f^{\prime}(t)\right)^{2} \geqq 0 .
$$

Using (2.1) we find that

$$
g(t)=\int_{0}^{\infty} \int_{0}^{\infty} \lambda(\lambda-\mu) e^{-(\lambda+\mu) t} d \phi(\lambda) d \phi(\mu) .
$$

Next,

$$
\begin{aligned}
\int_{0}^{\infty} \int_{\mu}^{\infty} \lambda(\lambda-\mu) e^{-(\lambda+\mu) t} d \phi(\lambda) d \phi(\mu) & =\int_{0}^{\infty} \int_{0}^{\lambda} \lambda(\lambda-\mu) e^{-(\lambda+\mu) t} d \phi(\mu) d \phi(\lambda) \\
& =\int_{0}^{\infty} \int_{0}^{\mu} \mu(\mu-\lambda) e^{-(\lambda+\mu) t} d \phi(\lambda) d \phi(\mu) .
\end{aligned}
$$

Therefore

$$
g(t)=\int_{0}^{\infty} \int_{0}^{\mu}(\cdots)+\int_{0}^{\infty} \int_{\mu}^{\infty}(\cdots)=\int_{0}^{\infty} \int_{0}^{\mu}(\lambda-\mu)^{2} e^{-(\lambda+\mu) t} d \phi(\lambda) d \phi(\mu) \geqq 0 .
$$

Definitions. A function $h(t)$ which belongs to $C(0, \infty)$ and to $L^{1}(0,1)$ is said to belong to the class $\mathscr{H}$, if $h(t) \geqq 0, h(t) \not \equiv 0$, and $h(t)$ is monotone nonincreasing in $(0, \infty)$. If $h \in \mathscr{H}$ and if $\log h(t)$ is a convex function in the interval where $h(t)>0$, then we say that $h$ belongs to the class $\mathscr{H}^{\prime}$. Finally, we say that $h \in \mathscr{H}_{\infty}$ if $h(t)$ is a nonzero completely monotonic function in $(0, \infty)$ and if $h \in L^{1}(0,1)$.

From Lemma 2.4 (applied to $h(t+\varepsilon)$, for any $\varepsilon>0$ ) it follows that if $h \in \mathscr{H}_{\infty}$ then $h \in \mathscr{H}^{\prime}$.

In the following lemma we have collected some results proved in Friedman [2].

LEMMA 2.5. Consider the integral equation

$$
x(t)=1-\int_{0}^{t} h(t-\tau) x(\tau) d \tau \quad(0<t<\infty) .
$$

(i) If $h \in \mathscr{H}$, then $0 \leqq x(t) \leqq 1$.

(ii) If $h \in \mathscr{H}^{\prime}$, then $x(t)$ is monotone nonincreasing.

(iii) If $h \in \mathscr{H}_{\infty}$, then $x(t)$ is in $\mathscr{H}_{\infty}$.

From [2, Corollary 4, p. 387] we deduce

LEMMA 2.6. Consider the equation

$$
x(t)=\int_{0}^{t} h(t-\sigma) p(\sigma) d \sigma-\lambda \int_{0}^{t} h(t-\tau) x(\tau) d \tau \quad(0<t<\infty)
$$

where $p(\sigma)$ is a continuous nonnegative function, and $\lambda$ is a positive constant. If $h \in \mathscr{H}^{\prime}$ then $x(t) \geqq 0$.

We shall consider now the equation

$$
S_{\lambda}(t)=1-\lambda \int_{0}^{t} h(t-\tau) S_{\lambda}(\tau) d \tau \quad(0 \leqq t<\infty)
$$


where $\lambda$ is a complex parameter. By a standard argument one shows that if $h(t)$ is in $C(0, \infty) \cap L^{1}(0,1)$ then, for each $\lambda$, there exists a unique solution $S_{\lambda}(t)$ of (2.4). Furthermore, $S_{\lambda}(t)$ is continuous in $(t, \lambda)(t \geqq 0, \lambda$ complex) and analytic in $\lambda$, for each $t \geqq 0$. If $h \in C^{n}[0, \infty)$ then $\partial^{n} S_{\lambda}(t) / \partial t^{n}$ is continuous in $(t, \lambda)$ (for $t \geqq 0, \lambda$ complex) and analytic in $\lambda$, for each $t \geqq 0$.

Lemma 2.7. If $h \in \mathscr{H}$ then, for $n=0,1,2, \ldots$,

$$
(-1)^{n}\left(\partial^{n} S_{\lambda}(t) / \partial \lambda^{n}\right) \geqq 0 \quad \text { if } 0<\lambda<\infty, 0<t<\infty .
$$

Proof. The inequality (2.5) for $n=0$ follows from Lemma 2.5(i). We proceed by induction. We assume that (2.5) holds and prove the same inequality when $n$ is replaced by $n+1$. Differentiating (2.4) $n+1$ times with respect to $\lambda$ we get

$$
\frac{\partial^{n+1}}{\partial \lambda^{n+1}} S_{\lambda}(t)=-(n+1) \int_{0}^{t} h(t-\tau) \frac{\partial^{n}}{\partial \lambda^{n}} S_{\lambda}(\tau) d \tau-\lambda \int_{0}^{t} h(t-\tau) \frac{\partial^{n+1}}{\partial \lambda^{n+1}} S_{\lambda}(\tau) d \tau .
$$

It follows that the function $x(t)=(-1)^{n+1} \partial^{n+1} S_{\lambda}(t) / \partial \lambda^{n+1}$ satisfies the equation (2.3) with

$$
p(\sigma)=(n+1)(-1)^{n}\left(\partial^{n} S_{\lambda}(\sigma) / \partial \lambda^{n}\right) .
$$

By the inductive assumption, $p(\sigma) \geqq 0$. Hence we can apply Lemma 2.6 and conclude that $x(t) \geqq 0$, i.e., $(2.5)$ holds with $n$ replaced by $n+1$.

Lemma 2.8. If $h \in \mathscr{H}^{\prime}$ then, for $n=0,1,2, \ldots, \lambda>0$,

$$
(-1)^{n} \frac{\partial^{n}}{\partial \lambda^{n}}\left[\frac{S_{\lambda}(t)}{\lambda}\right] \searrow \text { if } t \nearrow \text {. }
$$

Proof. We may assume that $h \in C^{1}[0, \infty)$. Indeed, otherwise we approximate $h(t)$ by a sequence of functions $\left\{h_{m}(t)\right\}$ as follows: $h_{m} \in \mathscr{H}^{\prime}, h_{m}(t) \rightarrow h(t)$ uniformly on compact subsets of $(0, \infty)$ and

$$
\int_{0}^{1}\left|h(t)-h_{m}(t)\right| d t \rightarrow 0 .
$$

If we know already that the assertion of the lemma holds for the solution $S_{\lambda, m}(t)$ corresponding to $h_{m}$, then (2.7) is also true since, for any $n \geqq 0$,

$$
\frac{\partial^{n}}{\partial \lambda^{n}}\left[\frac{S_{\lambda, m}(t)}{\lambda}\right] \rightarrow \frac{\partial^{n}}{\partial \lambda^{n}}\left[\frac{S_{\lambda}(t)}{\lambda}\right] \text { for each } t
$$

Assuming $h$ to be in $C^{1}[0, \infty)$, it follows that $\partial S_{\lambda}(t) / \partial t$ exists and satisfies:

$$
\frac{\partial S_{\lambda}(t)}{\partial t}=-\lambda h(t)-\lambda \int_{0}^{t} h(t-\tau) \frac{\partial S_{\lambda}(\tau)}{\partial \tau} d \tau
$$

The assertion (2.7) is equivalent to the following inequality:

$$
(-1)^{n+1} \frac{\partial^{n}}{\partial \lambda^{n}}\left[\frac{1}{\lambda} \frac{\partial}{\partial t} S_{\lambda}(t)\right] \geqq 0
$$


For $n=0$, this inequality follows from Lemma 2.5(ii). We now proceed by induction on $n$. To pass from $n$ to $n+1$, we divide both sides of (2.8) by $\lambda$ and then differentiate both sides $n+1$ times with respect to $\lambda$. We get

$$
\begin{aligned}
\frac{\partial^{n+1}}{\partial \lambda^{n+1}}\left[\frac{1}{\lambda} \frac{\partial S_{\lambda}(t)}{\partial t}\right]= & -(n+1) \int_{0}^{t} h(t-\tau) \frac{\partial^{n}}{\partial \lambda^{n}}\left[\frac{1}{\lambda} \frac{\partial S_{\lambda}(\tau)}{\partial \tau}\right] d \tau \\
& -\lambda \int_{0}^{t} h(t-\tau) \frac{\partial^{n+1}}{\partial \lambda^{n+1}}\left[\frac{1}{\lambda} \frac{\partial S_{\lambda}(\tau)}{\partial \tau}\right] d \tau
\end{aligned}
$$

We can now apply Lemma 2.6 with

$$
x(t)=(-1)^{n+2} \frac{\partial^{n+1}}{\partial \lambda^{n+1}}\left[\frac{1}{\lambda} \frac{\partial S_{\lambda}(t)}{\partial t}\right], \quad p(\sigma)=(n+1)(-1)^{n+1} \frac{\partial^{n}}{\partial \lambda^{n}}\left[\frac{1}{\lambda} \frac{\partial S_{\lambda}(\sigma)}{\partial \sigma}\right] .
$$

Lemma 2.9. If $h \in \mathscr{H}_{\infty}$ then, for $n=0,1,2, \ldots, m=0,1,2, \ldots, \lambda>0$,

$$
(-1)^{m+n} \frac{\partial}{\partial \lambda^{m}}\left[\frac{1}{\lambda^{n}} \frac{\partial^{n}}{\partial t^{n}} S_{\lambda}(t)\right] \geqq 0 \text { for } t>0 .
$$

Proof. Suppose first that $h(t)$ is in $C^{\infty}[0, \infty)$. Then all the derivatives occurring in (2.10) exist for $t \geqq 0$. We shall establish (2.10) by induction on $n$. For $n=0,(2.10)$ follows from Lemma 2.7. We now assume that (2.10) holds for all $m \geqq 0$ and $0 \leqq n$ $\leqq k$. We shall prove $(2.10)$ for all $m \geqq 0$ and $n=k+1$. Differentiating both sides of (2.4) $k+1$ times with respect to $t$, we get

$$
\begin{aligned}
\frac{\partial^{k+1} S_{\lambda}(t)}{\partial t^{k+1}}= & -\lambda h^{(k)}(t) S_{\lambda}(0)-\lambda h^{(k-1)}(t) \frac{\partial S_{\lambda}(0)}{\partial t}-\cdots-\lambda h(t) \frac{\partial^{k} S_{\lambda}(0)}{\partial t^{k}} \\
& -\lambda \int_{0}^{t} h(t-\tau) \frac{\partial^{k+1} S_{\lambda}(\tau)}{\partial \tau^{k+1}} d \tau .
\end{aligned}
$$

Setting

$$
T_{\lambda}(t)=(-1)^{k+1} \frac{1}{\lambda^{k+1}} \frac{\partial^{k+1}}{\partial t^{k+1}} S_{\lambda}(t)
$$

we get

$$
\begin{aligned}
T_{\lambda}(t)= & \sum_{i=0}^{k} \frac{(-1)^{k-i} h^{(k-i)}(t)}{\lambda^{k-i}} \frac{(-1)^{i} \partial^{i} S_{\lambda}(0) / \partial t^{i}}{\lambda^{i}} \\
& -\lambda \int_{0}^{t} h(t-\tau) T_{\lambda}(\tau) d \tau
\end{aligned}
$$

We have to prove that, for any $m \geqq 0$,

$$
(-1)^{m}\left(\partial^{m} T_{\lambda}(t) / \partial \lambda_{m}\right) \geqq 0 .
$$

For $m=0$ this follows from the definition of $T_{\lambda}(t)$ and Lemma 2.5(iii). We now proceed by induction on $m$.

To pass from $m$ to $m+1$, we differentiate (2.11) $m+1$ times with respect to $\lambda$. 
We find

$$
\begin{aligned}
\frac{\partial^{m+1} T_{\lambda}(t)}{\partial \lambda^{m+1}}= & g(t)-(m+1) \int_{0}^{t} h(t-\tau) \frac{\partial^{m} T_{\lambda}(\tau)}{\partial \lambda^{m}} d \tau \\
& -\lambda \int_{0}^{t} h(t-\tau) \frac{\partial^{m+1} T_{\lambda}(\tau)}{\partial \lambda^{m+1}} d \tau
\end{aligned}
$$

where

$$
\begin{aligned}
& g(t)=\sum_{i=0}^{k} g_{i}(t) \\
& g_{i}(t)=(-1)^{k-i} h^{(k-i)}(t) \sum_{s=0}^{m+1}\left(\begin{array}{c}
m+1 \\
s
\end{array}\right) \frac{d^{s}}{d \lambda^{s}} \frac{1}{\lambda^{k-i}} \cdot \frac{\partial^{m+1-s}}{\partial \lambda^{m+1-s}}\left[\frac{(-1)^{i}}{\lambda^{i}} \frac{\partial^{i}}{\partial t^{i}} S_{\lambda}(0)\right] .
\end{aligned}
$$

Set

$$
g_{k+1}(t)=-(m+1) \int_{0}^{t} h(t-\tau) \frac{\partial^{m} T_{\lambda}(\tau)}{\partial \lambda^{m}} d \tau
$$

and denote by $x_{i}(t)(0 \leqq i \leqq k+1)$ the solution of the equation

$$
x_{i}(t)=(-1)^{m+1} g_{i}(t)-\lambda \int_{0}^{t} h(t-\tau) x_{i}(\tau) d \tau .
$$

It is clear that

$$
(-1)^{m+1} \frac{\partial^{m+1} T_{\lambda}(t)}{\partial \lambda^{m+1}}=\sum_{i=0}^{k+1} x_{i}(t)
$$

Hence, it suffices to show that $x_{i}(t) \geqq 0$ for $0 \leqq i \leqq k+1$.

The inequality $x_{k+1}(t) \geqq 0$ follows by applying Lemma 2.6 with

$$
p(\sigma)=(-1)^{m}(m+1) \partial^{m} T_{\lambda}(\sigma) / \partial \lambda^{m} ;
$$

note that by the inductive assumption, $p(\sigma) \geqq 0$.

From (2.14) and the inductive assumption we easily see that

$$
(-1)^{m+1} g_{i}(t)=\gamma_{i}(-1)^{k-i} h^{(k-i)}(t) \quad(0 \leqq i \leqq k)
$$

where $\gamma_{i}=\gamma_{i}(\lambda)$ is nonnegative. From Theorem 1 and its Corollary 3 in [2] we then have the following: If

$$
\frac{h^{\prime}(a)}{h(a)} \leqq \frac{h^{(k-i+1)}(b)}{h^{(k-i)}(b)} \quad(0<a<b<\infty)
$$

then $x_{i}(t) \geqq 0$. Thus, it remains to prove (2.17). We assume here that $h^{(k-i)}(b)>0$ for all $b>0$; if $h^{(k-i)}(b)=0$ for some $b>0$ then $h^{(k-i)}(t) \equiv 0$ and $x_{i}(t) \equiv 0$.

Since $h \in \mathscr{H}_{\infty}$, Lemma 2.4 implies that $h^{\prime}(t) / h(t) \nearrow$ if $t \nearrow$. Hence (2.17) is a consequence of

$$
\frac{h^{\prime}(b)}{h(b)} \leqq \frac{h^{(k-i+1)}(b)}{h^{(k-i)}(b)} \quad(0<b<\infty)
$$


Now, the function $(-1)^{j} h^{(j)}(t)$ is completely monotonic. If we apply Lemma 2.4 to this function, we obtain

$$
h^{(j+1)}(b) / h^{(j)}(b) \leqq h^{(j+2)}(b) / h^{(j+1)}(b) .
$$

Applying this inequality for $j=0,1, \ldots, k-i-1$, we get (2.18).

We have proved Lemma 2.9 assuming that $h(t)$ is in $C^{\infty}[0, \infty)$. Consider now the general case, where we merely assume that $h \in \mathscr{H}_{\infty}$. Then we can apply the previous result to the solution $S_{\lambda, \varepsilon}(t)$ of (2.4) with $h(t)$ replaced by $h(t+\varepsilon), \varepsilon>0$. Since, for each $t>0, m \geqq 0$,

$$
\partial^{m} S_{\lambda, \varepsilon}(t) / \partial \lambda^{m} \rightarrow \partial^{m} S_{\lambda}(t) / \partial \lambda^{m}
$$

the assertion of the lemma for $S_{\lambda}(t)$ follows upon applying Lemma 2.3.

The last lemma of this section is the following:

Lemma 2.10. Let $h \in \mathscr{H}$. Then, for $n=0,1,2, \ldots$,

$$
(-1)^{n}\left(\partial^{n} S_{\lambda}(t) / \partial \lambda^{n}\right) \leqq n ! / \lambda^{n} \quad(0<\lambda<\infty, 0<t<\infty) .
$$

Proof. From Lemma 2.7 and (2.6) we obtain

$$
(-1)^{n+1} \int_{0}^{t} h(t-\tau) \frac{\partial^{n+1}}{\partial \lambda^{n+1}} S_{\lambda}(\tau) d \tau \leqq(-1)^{n} \frac{n+1}{\lambda} \int_{0}^{t} h(t-\tau) \frac{\partial^{n}}{\partial \lambda^{n}} S_{\lambda}(\tau) d \tau .
$$

Applying this relation successively, we find that

$$
(-1)^{m} \int_{0}^{t} h(t-\tau) \frac{\partial^{m}}{\partial \lambda^{m}} S_{\lambda}(\tau) d \tau \leqq \frac{m !}{\lambda^{m+1}} \quad(m=0,1,2, \ldots) .
$$

Hence from (2.6), with $n+1=m$, we get

$$
(-1)^{m} \frac{\partial^{m}}{\partial \lambda^{m}} S_{\lambda}(t) \leqq(-1)^{m-1} m \int_{0}^{t} h(t-\tau) \frac{\partial^{m-1}}{\partial \lambda^{m-1}} S_{\lambda}(\tau) d \tau \leqq \frac{m !}{\lambda^{m}}
$$

for $m=1,2, \ldots$

3. Integral formula for $S(t)$. Let $X$ be a Banach space. We denote by $\sigma(A)$ the spectrum of an operator $A$.

THEOREM 3.1. Let $A$ be a bounded operator and let $\Gamma$ be any continuously differentiable closed Jordan curve containing $\sigma(A)$ in its interior. Let $h(t)$ be any function in $C(0, \infty) \cap L^{1}(0,1)$. Then the operator-valued function

$$
S(t) \equiv \frac{1}{2 \pi i} \int_{\Gamma}(\lambda I-A)^{-1} S_{\lambda}(t) d \lambda
$$

is the unique fundamental solution of (1.1).

The orientation of $\Gamma$, in (3.1), is taken counterclockwise.

Proof. The uniqueness of the fundamental solution follows by standard arguments. It remains to verify (1.2). We have 


$$
\begin{aligned}
T & \equiv I-A \int_{0}^{t} h(t-\tau) S(\tau) d \tau \\
& =I-\int_{0}^{t} h(t-\tau)\left\{\frac{1}{2 \pi i} \int_{\Gamma} A(\lambda I-A)^{-1} S_{\lambda}(\tau) d \lambda\right\} d \tau .
\end{aligned}
$$

Changing the order of integration and using (2.4), we get

$$
\begin{aligned}
T & =I+\frac{1}{2 \pi i} \int_{\Gamma}\left[(\lambda I-A)^{-1}-\frac{I}{\lambda}\right]\left[S_{\lambda}(t)-1\right] d \lambda \\
& =I+S(t)-\frac{1}{2 \pi i} \int_{\Gamma}(\lambda I-A)^{-1} d \lambda+\frac{I}{2 \pi i} \int_{\Gamma} \frac{1-S_{\lambda}(t)}{\lambda} d \lambda .
\end{aligned}
$$

By Cauchy's theorem we easily find that

$$
\frac{1}{2 \pi i} \int_{\Gamma}(\lambda I-A)^{-1} d \lambda=I
$$

Also,

$$
\begin{aligned}
\int_{\Gamma} \frac{1-S_{\lambda}(t)}{\lambda} d \lambda & =\int_{\Gamma}\left\{\int_{0}^{t} h(t-\tau) S_{\lambda}(\tau) d \tau\right\} d \lambda \\
& =\int_{0}^{t} h(t-\tau)\left\{\int_{\Gamma} S_{\lambda}(\tau) d \lambda\right\} d \tau=0
\end{aligned}
$$

We obtain that $T=S(t)$. This proves (1.2).

Theorem 3.1 can easily be extended to more general integral equations. For example, we shall construct a solution of

$$
S(t, s)=I-\int_{s}^{t} h(t-\tau, \tau) A S(\tau, s) d \tau .
$$

Denote by $S_{\lambda}(t, s)$ the solution of

$$
S_{\lambda}(t, s)=1-\lambda \int_{s}^{t} h(t-\tau, \tau) S_{\lambda}(\tau, s) d \tau
$$

Then we have

THEOREM 3.1'. Let $A, \Gamma$ be as in Theorem 3.1 and let $h(t, \tau)$ be a continuous function for $t \geqq 0, \tau \geqq 0$. Then the unique solution of (3.2) is given by

$$
S(t, \tau)=\frac{1}{2 \pi i} \int_{\Gamma}(\lambda I-A)^{-1} S_{\lambda}(t, \tau) d \lambda .
$$

The proof is similar to the proof of Theorem 3.1.

We next consider the case where $A$ is not necessarily a bounded operator.

Definition. A linear operator $A$ in $X$ is said to belong to the class $\mathfrak{A}$ if

(i) $A$ is closed and densely defined;

(ii) $\sigma(A) \subset\left\{\lambda ;|\arg \lambda| \leqq \pi / 2-\varepsilon, \operatorname{Re} \lambda \geqq \lambda_{0}\right\}$ for some $\varepsilon>0, \lambda_{0}>0$;

(iii) $\left\|(\lambda I-A)^{-1}\right\| \leqq c /|\lambda|$ if $|\arg \lambda|>\pi / 2-\varepsilon$. 
Definition. A complex-valued function $h(t)$ is said to belong to the class $\mathscr{K}$ if

(i) $h(0)>0$;

(ii) $h \in C^{1}[0, \infty)$ and $h(t)$ is absolutely continuous;

(iii) for any $b>0, \tilde{h}(t)$ is in $L^{p}(0, b)$ for some $p>1$.

In [6, Chapter 1] it was proved that if $A \in \mathfrak{A}$ and $h \in \mathscr{K}$, then there exists a fundamental solution $W(t)$ of (1.1), in a sense different than (1.2). Thus, $W(t)$ satisfies the equation

$$
W(t)=I-\int_{0}^{t} h(t-\tau) A W(\tau) d \tau
$$

in the following sense

$$
\tilde{W}(t)=e^{-t A}+\int_{0}^{t} e^{-(t-\tau) A} F(W ; \tau) d \tau
$$

where $e^{-t A}$ is the analytic semigroup of $-A$, and

$$
\begin{gathered}
\tilde{W}(t)=W(t)+\frac{1}{h(0)} \int_{0}^{t} h(t-\tau) W(\tau) d \tau, \\
F(W ; \tau)=\frac{h(0)}{h(0)} W(t)+\frac{1}{h(0)} \int_{0}^{t} \ddot{h}(t-\tau) W(\tau) d \tau .
\end{gathered}
$$

Denoting by $D\left(A^{\mu}\right)$ the domain of $A^{\mu}$ (see [7] for the definition of $A^{\mu}$ ), we have the following result: If $x_{0} \in D\left(A^{\mu}\right)$ for some $\mu>0$, then $W(t) x_{0}$ is the unique solution of (1.1). (The solutions of (1.1) are assumed to be such that $\|A x(\tau)\|$ is integrable in every bounded interval $(0, b)$.)

We introduce the operators

$$
A_{n}=A(I+A / n)^{-1} .
$$

One easily verifies that $\left\|A_{n}\right\| \leqq C n$ and

$$
\left(\lambda I-A_{n}\right)^{-1}=-\frac{1}{n-\lambda} I+\frac{n^{2}}{(n-\lambda)^{2}}\left[\frac{n \lambda}{n+\lambda} I-A\right]^{-1} \text {. }
$$

Denote by $\Gamma_{n}$ a continuously differentiable closed Jordan curve which contains $\sigma\left(A_{n}\right)$, and set

$$
S^{n}(t)=\frac{1}{2 \pi i} \int_{\Gamma_{n}}\left(\lambda I-A_{n}\right)^{-1} S_{\lambda}(t) d \lambda .
$$

TheOREM 3.2. Let $h \in \mathscr{K}, A \in \mathfrak{A}$. Then, for any $x_{0} \in X$,

$$
\lim _{n \rightarrow \infty} S^{n}(t) x_{0}=W(t) x_{0}
$$

uniformly with respect to $t$ in bounded intervals $[0, b)$. 
Proof. Suppose first that $x_{0} \in D(A)$. Set

$$
\begin{aligned}
& u_{n}(t)=S^{n}(t) x_{0}, \quad u(t)=W(t) x_{0}, \\
& \tilde{u}_{n}(t)=u_{n}(t)+\frac{1}{h(0)} \int_{0}^{t} h(t-\tau) u_{n}(\tau) d \tau, \\
& \tilde{u}(t)=u(t)+\frac{1}{h(0)} \int_{0}^{t} h(t-\tau) u(\tau) d \tau
\end{aligned}
$$

To prove (3.12) it suffices to show that

$$
\lim _{n \rightarrow \infty} \tilde{u}_{n}(t)=\tilde{u}(t)
$$

uniformly in $t$ in bounded intervals $[0, b)$.

From [6] we have

$$
\tilde{u}(t)=e^{-t A} x_{0}+\int_{0}^{t} e^{-(t-\tau) A}\left[\frac{h(0)}{h(0)} u(\tau)+\frac{1}{h(0)} \int_{0}^{\tau} \ddot{h}(\tau-s) u(s) d s\right] d \tau .
$$

Similarly,

$$
\tilde{u}_{n}(t)=e^{-t A_{n}} x_{0}+\int_{0}^{t} e^{-(t-\tau) A_{n}}\left[\frac{h(0)}{h(0)} u_{n}(\tau)+\frac{1}{h(0)} \int_{0}^{\tau} \tilde{h}(\tau-s) u_{n}(s) d s\right] d \tau
$$

From the definition of $e^{-t A}$ (see [7]) as an integral of the form

$$
\frac{1}{2 \pi i} \int_{C} e^{\lambda t}(\lambda I+A)^{-1} d \lambda
$$

and from the relation

$$
\left\|\left[A_{n}-A\right] A^{-1} y_{0}\right\| \rightarrow 0 \text { as } n \rightarrow \infty\left(\text { for any } y_{0} \in X\right)
$$

we find that, for any continuous function $v(s)$,

$$
\lim _{n \rightarrow \infty}\left\|\left[e^{-t A}-e^{-t A_{n}}\right] A^{-1} v(s)\right\|=0
$$

uniformly with respect to $t, s$ in bounded sets of $[0, \infty)$.

Subtracting (3.14) from (3.15) and using (3.17) with $v(t)=A x_{0}$ and with $v(t)$ $=A u(t)$, we get

$$
\left\|\tilde{u}(t)-\tilde{u}_{n}(t)\right\| \leqq C(t) \int_{0}^{t}\left\|\tilde{u}(\tau)-\tilde{u}_{n}(\tau)\right\| d \tau+\varepsilon_{n}(t)
$$

where $C(t)$ is bounded in bounded intervals $[0, b)$, and $\varepsilon_{n}(t) \rightarrow 0$, as $n \rightarrow \infty$, uniformly in $t$ in bounded intervals [0,b). The last inequality gives (3.13).

Having proved (3.12) for $x_{0} \in D(A)$, we next notice that, in any bounded interval $0 \leqq t \leqq b,\left\|S^{n}(t)\right\| \leqq C$ where $C$ is a constant independent of $n, t$. In fact, since (3.6)-(3.8) hold for $A=A_{n}, W=S^{n}$, the latter bound follows from the estimates on $W$ obtained in [6, Chapter 1]. It follows that (3.12) holds for all $x_{0} \in X$, uniformly in $t$ in bounded intervals. 
Remark. A result similar to Theorem 3.2 holds also with respect to the more general integral equation (3.2).

Definition. A complex-valued function $h(t)$ is said to belong to the class $\mathscr{K}^{\prime}$ if

(i) $h(0)>0$ and $h(t)$ is absolutely continuous in $[0, \infty)$;

(ii) $h(t) \in L^{1}(0, \infty)$.

If $h \in \mathscr{K}^{\prime}$ then we can introduce the function

$$
g(s)=h(0)+h^{\wedge}(s) \text { for } \operatorname{Re} s \geqq 0,
$$

where $f^{\wedge}(s)$ indicates the Laplace transform of $f(t)$. Then $g(s)=s h^{\wedge}(s)$ if $\operatorname{Re} s>0$. It follows that $h^{\wedge}(s)$ can be defined by continuity for $\operatorname{Re} s \geqq 0, s \neq 0$. If $g(0) \neq 0$, then we let $h^{\wedge}(0)=\infty$, and introduce the set

$$
\Delta \equiv\left\{-1 / h^{\wedge}(s) ; \operatorname{Re} s \geqq 0\right\} .
$$

As proved in [6, Chapter 2], if $h \in \mathscr{K}^{\prime}, A \in \mathfrak{A}$, and if

$$
g(s) \neq 0 \text { for all } s \text { with } \operatorname{Re} s \geqq 0,
$$

$$
\Delta \subset \rho(A),
$$

then there exists a fundamental solution $S(t)$ of (1.1) in the sense defined in $\S 1$ (cf. (1.2)), and it belongs to $L^{p}(0, \infty ; B(X))$ for any $p \geqq 2$.

Analogously to Theorem 3.2 , we have

THeOREM 3.3. L'et $h \in \mathscr{K}^{\prime}, A \in \mathfrak{A}$, and let (3.20), (3.21) hold. Then for any $p \geqq 2$, and for any $x_{0} \in X$,

$$
\lim _{n \rightarrow \infty} \int_{0}^{\infty}\left\|S^{n}(t) x_{0}-S(t) x_{0}\right\|^{p} d t=0 .
$$

Proof. In [6, Chapter 2] it was proved that

$$
S(t)=\frac{1}{2 \pi i} \int_{C}(\lambda I-A)^{-1} S_{\lambda}(t) d \lambda
$$

for an appropriate curve $C$ lying in the resolvent set $\rho(A)$ of $A$, where $S_{\lambda}(t)$ is the inverse Laplace transform of the function

$$
1 /(s+\lambda g(s)) \text {. }
$$

One can easily verify that if $S_{\lambda}(t)$ is the solution of (2.4) then its Laplace transform coincides with the function (3.24). Hence, by the uniqueness of the inverse Laplace transform we conclude that the function $S_{\lambda}(t)$ occurring in (3.23) coincides with the solution of (2.4).

Using the definition of $S^{n}(t)$ in (3.11) and Cauchy's theorem, we have:

$$
S^{n}(t)=\frac{1}{2 \pi i} \int_{C}\left(\lambda I-A_{n}\right)^{-1} S_{\lambda}(t) d t .
$$


Noting that

$$
\left\|\left(\lambda I-A_{n}\right)^{-1} x_{0}-(\lambda I-A)^{-1} x_{0}\right\| \leqq\left\|\left(\lambda I-A_{n}\right)^{-1}\right\|\left\|\left(A_{n}-A\right)(\lambda I-A)^{-1} x_{0}\right\| \leqq \varepsilon_{n} /|\lambda|
$$

where $\varepsilon_{n} \rightarrow 0$ if $n \rightarrow \infty$, we obtain from (3.23), (3.25):

Since, by [6],

$$
\left\|S^{n}(t) x_{0}-S(t) x_{0}\right\| \leqq c \varepsilon_{n} \int_{C}\left|S_{\lambda}(t)\right| \frac{|d \lambda|}{|\lambda|} .
$$

we obtain

$$
\left\{\int_{0}^{\infty}\left|S_{\lambda}(t)\right|^{p} d t\right\}^{1 / p} \leqq \frac{c}{|\lambda|^{1 / p}} \quad \text { if } p \geqq 2
$$

$$
\left\{\int_{0}^{\infty}\left\|S^{n}(t) x_{0}-S(t) x_{0}\right\|^{p} d t\right\}^{1 / p} \leqq c \varepsilon_{n} \int_{C} \frac{|d \lambda|}{|\lambda|^{1+1 / p}} \rightarrow 0
$$

as $n \rightarrow \infty$. This proves (3.22).

4. Monotonicity for $A$ selfadjoint. Let $X$ be a Hilbert space and let $A$ be a selfadjoint operator in $X$. We say that $A$ is strictly positive if the number

$$
\delta_{A}=\inf _{x \neq 0} \frac{(A x, x)}{(x, x)}
$$

is positive. The main result of the present section is the following:

THEOREM 4.1. Let $X$ be a Hilbert space and let $A$ be a strictly positive selfadjoint operator, with the spectral decomposition of the identity $\left\{E_{\lambda}\right\}$. If $h \in \mathscr{H}$ then the operator $S(t)$ given by

$$
S(t) x_{0}=\int_{\delta_{A}}^{\infty} S_{\mu}(t) d E_{\mu} x_{0} \quad\left(x_{0} \in X\right)
$$

is a fundamental solution of (1.1).

Note that (4.1) is formally obtained from (3.1) and the formula

$$
(\lambda I-A)^{-1}=\int_{\delta_{A}}^{\infty} \frac{d E_{\mu}}{\lambda-\mu}
$$

using the Cauchy formula.

Proof. Since $0 \leqq S_{\mu}(t) \leqq 1$, the integral in (4.1) exists and $\left\|S(t) x_{0}\right\| \leqq\left\|x_{0}\right\|$. $S(t) x_{0}$ is clearly continuous in $t$. Next, by Fubini's theorem,

$$
\int_{0}^{t} h(t-\tau) S(\tau) x_{0} d \tau=\int_{\delta_{A}}^{\infty}\left\{\int_{0}^{t} h(t-\tau) S_{\mu}(\tau) d \tau\right\} d E_{\mu} x_{0} .
$$

Using (2.4) we find the expression on the right is equal to

$$
\int_{\delta_{A}}^{\infty}\left\{\frac{1}{\mu}-\frac{S_{\mu}(t)}{\mu}\right\} d E_{\mu} x_{0}=A^{-1} x_{0}-A^{-1} S(t) x_{0},
$$


where (4.1) has been used. We have thus proved that

$$
\int_{0}^{t} h(t-\tau) S(\tau) x_{0} d \tau
$$

lies in $D(A)$ and that if we apply $A$ to this integral we obtain $x_{0}-S(t) x_{0}$. This completes the proof of (1.2).

From Theorem 4.1 and Lemma 2.5(i), (ii) we obtain

Corollary 1. For any $x_{0} \in X$,

$$
0 \leqq\left(S(t) x_{0}, x_{0}\right) \leqq\left\|x_{0}\right\|^{2} \quad(0<t<\infty) .
$$

Corollary 2. If $h \in \mathscr{H}^{\prime}$ then, for any $x_{0} \in X$,

$$
\left(S(t) x_{0}, x_{0}\right) \searrow \text { if } t \nearrow \quad(0<t<\infty) .
$$

If $S_{\mu}(t) \searrow$ when $t \nearrow(\mu>0)$, then we obtain from (2.4) the bound

$$
S_{\mu}(t) \leqq\left[1+\mu \int_{0}^{t} h(\tau) d \tau\right]^{-1}
$$

We conclude

CoRollary 3. If $h \in \mathscr{H}^{\prime}$ then, for any $x_{0} \in X$,

$$
\left(S(t) x_{0}, x_{0}\right) \leqq \int_{\delta_{A}}^{\infty}\left[1+\mu \int_{0}^{t} h(\tau) d \tau\right]^{-1} d\left(E_{\mu} x_{0}, x_{0}\right)
$$

We next have

Corollary 4. If $h \in \mathscr{H}_{\infty}$ then, for any $x_{0} \in X$,

$$
(-1)^{n} \frac{d^{n}}{d t^{n}}\left(S(t) x_{0}, x_{0}\right) \geqq 0 \quad(n=0,1,2, \ldots ; 0<t<\infty) .
$$

Proof. By Lemma 2.5(iii), the functions

$$
T_{m}(t)=\int_{\delta_{A}}^{m+\delta_{A}} S_{\mu}(t) d\left(E_{\mu} x_{0}, x_{0}\right) \quad(m=1,2, \ldots)
$$

are completely monotonic in $(0, \infty)$. Since, for each $t>0, T_{m}(t) \rightarrow\left(S(t) x_{0}, x_{0}\right)$ as $m \rightarrow \infty$, the assertion of the corollary follows from Lemma 2.3.

REMARK. Theorem 4.1 extends, with the same proof, to the case of the integral equation (3.2).

\section{Monotonicity for general $A$.}

Definition. A closed linear operator $A$ with a dense domain is said to belong to the class $\mathfrak{A}^{\prime}$ if it satisfies the following properties:

(i) $(\lambda I-A)^{-1}$ exists for all complex $\lambda$, except for a sequence $\left\{\mu_{k}\right\}$ (which may be finite) of positive and increasing numbers with no finite limit.

(ii) At each $\mu_{k},(\lambda I-A)^{-1}$ has a pole, i.e.,

$$
(\lambda I-A)^{-1}=\sum_{j=1}^{m_{k}} \frac{B_{k, j}}{\left(\lambda-\mu_{k}\right)^{j}}+B_{k, 0}(\lambda)
$$


where $B_{k, j}$ are bounded operators and $B_{k, 0}(\lambda)$ is an analytic function (with values in $B(X)$ ) in a neighborhood of $\lambda=\mu_{k}$.

From (3.1), (5.1) and the residue theorem, we formally obtain the formula

$$
S(t) x_{0}=\sum_{k=1}^{\infty} \sum_{j=1}^{m_{k}} \frac{1}{(j-1) !} \frac{\partial^{j-1} S_{\mu_{k}}(t)}{\partial \mu^{j-1}} B_{k, j} x_{0} .
$$

(We consider here the case where $\left\{\mu_{k}\right\}$ is an infinite sequence; the modifications for the case of a finite sequence are trivial.)

To show that $S(t)$ is a fundamental solution (under certain assumptions), we introduce the operators $T_{p}(t)$ defined by

$$
T_{p}(t)=\sum_{k=1}^{p} \sum_{j=1}^{m_{k}} \frac{1}{(j-1) !} \frac{\partial^{j-1} S_{\mu_{k}}(t)}{\partial \mu^{j-1}} B_{k, j}
$$

and set

$$
\Delta_{p}(t)=-T_{p}(t)+I-A \int_{0}^{t} h(t-\tau) T_{p}(\tau) d \tau
$$

Applying $\lambda I-A=\left(\lambda-\mu_{k}\right) I+\left(\mu_{k} I-A\right)$ to both sides of (5.1), we obtain the relations:

$$
\begin{aligned}
A B_{k, m_{k}}-\mu_{k} B_{k, m_{k}} & =0, \\
A B_{k, j}-\mu_{k} B_{k, j} & =B_{k, j+1} \quad\left(1 \leqq j \leqq m_{k}-1\right) .
\end{aligned}
$$

Using these relations and (2.6), (2.4), we get

$$
\begin{aligned}
\Delta_{p}(t)= & -\sum_{k=1}^{p} \sum_{j=1}^{m_{k}} \frac{1}{(j-1) !} \frac{\partial^{j-1} S_{\mu_{k}}(t)}{\partial \mu^{j-1}} B_{k, j}+I \\
& -\int_{0}^{t} h(t-\tau) \sum_{k=1}^{p} \sum_{j=1}^{m_{k}} \frac{1}{(j-1) !} \frac{\partial^{j-1} S_{\mu_{k}}(t)}{\partial \mu^{j-1}} \mu_{k} B_{k, j} d \tau \\
& -\int_{0}^{t} h(t-\tau) \sum_{k=1}^{p} \sum_{j=1}^{m_{k}-1} \frac{1}{(j-1) !} \frac{\partial^{j-1} S_{\mu_{k}}(t)}{\partial \mu^{j-1}} B_{k, j+1} d \tau \\
= & I-\sum_{k=1}^{p} B_{k, 1} .
\end{aligned}
$$

Definition. We denote by $X_{A}$ the set of all elements $x_{0}$ of $X$ for which

$$
\begin{aligned}
& \sum_{k=1}^{\infty} \sum_{j=1}^{m_{k}} \frac{\left\|B_{k, j} x_{0}\right\|}{\mu_{k}^{j-1}}<\infty, \\
& \lim _{p \rightarrow \infty} \sum_{k=1}^{p} B_{k, 1} x_{0}=x_{0} .
\end{aligned}
$$

TheOREM 5.1. Let $h \in \mathscr{H}, A \in \mathfrak{A}^{\prime}, x_{0} \in X_{A}$. Then the limit

$$
T(t) x_{0} \equiv \lim _{p \rightarrow \infty} T_{p}(t) x_{0}
$$


exists uniformly with respect to $t, 0 \leqq t<\infty$, and

$$
T(t) x_{0}=x_{0}-A \int_{0}^{t} h(t-\tau) T(\tau) x_{0} d \tau .
$$

Proof. The uniform convergence of the sequence $\left\{T_{p}(t) x_{0}\right\}$ follows from Lemma 2.10 and the assumption (5.7). From (5.6) we have

$$
T_{p}(t) x_{0}=x_{0}-A \int_{0}^{t} h(t-\tau) T_{p}(\tau) x_{0} d \tau-\Delta_{p}(t) x_{0}
$$

where

$$
\Delta_{p}(t) x_{0}=x_{0}-\sum_{k=1}^{p} B_{k, 1} x_{0}
$$

By (5.8), $\Delta_{p}(t) x_{0} \rightarrow 0$ as $p \rightarrow \infty$. Hence, taking $p \rightarrow \infty$ in (5.11) and using the assumption that $A$ is a closed operator, we conclude that the integral

$$
\int_{0}^{t} h(t-\tau) T(\tau) x_{0} d \tau
$$

belongs to the domain of $A$ and that (5.10) holds.

COROLlaRY 1. If, in addition to the assumptions of Theorem 5.1, we assume that $x_{0} \in D(A)$ and $A x_{0} \in X_{A}$, then

$$
T(t) x_{0}=x_{0}-\int_{0}^{t} h(t-\tau) A T(\tau) x_{0} d \tau
$$

and $T(t) x_{0}$ is continuous for $t \geqq 0$.

Proof. We have

$$
T_{p}(t) x_{0}=x_{0}-\int_{0}^{t} h(t-\tau) A T_{p}(\tau) x_{0} d \tau-\Delta_{p}(t) x_{0},
$$

and $A T_{p}(\tau) x_{0}=T_{p}(\tau)\left(A x_{0}\right)$. Since $A x_{0} \in X_{A}, T_{p}(\tau)\left(A x_{0}\right) \rightarrow T(\tau)\left(A x_{0}\right)$ as $p \rightarrow \infty$, uniformly with respect to $\tau$. It follows that $T(\tau) x_{0}$ is in $D(A)$, and $A T(\tau) x_{0}=T(\tau)\left(A x_{0}\right)$. Now take $p \rightarrow \infty$ in (5.13).

Corollary 2. Let $h \in \mathscr{H} \cap \mathscr{K}, A \in \mathfrak{A} \cap \mathfrak{A}^{\prime}, x_{0} \in X_{A}, A x_{0} \in X_{A}$. Then

$$
T(t) x_{0}=W(t) x_{0}
$$

where $W(t)$ is the fundamental solution of (1.1) occurring in Theorem 3.2.

Indeed, both sides of (5.14) are solutions of (1.1). By the uniqueness assertion of [6, Theorem 1], they must coincide.

Corollary 3. Let $h \in \mathscr{H} \cap \mathscr{K}^{\prime}, A \in \mathfrak{A} \cap \mathfrak{A}^{\prime}, x_{0} \in X_{A}$, and assume also that $\operatorname{th}(t) \in L^{1}(0, \infty)$, and that (3.20), (3.21) hold. Then

$$
T(t) x_{0}=S(t) x_{0}
$$

where $S(t)$ is the fundamental solution of (1.1) occurring in Theorem 3.3. 
This follows from (5.10) and the uniqueness assertion of [6, Theorem 10].

We recall [6] that if $h \in \mathscr{H}$ then the condition (3.20) is equivalent to the condition

$$
h(\infty)>0 .
$$

We shall now study monotonicity of the scalar function $f_{0}\left(T(t) x_{0}\right)$, where $f_{0}$ is any bounded linear functional in $X$.

Theorem 5.2. Let $h \in \mathscr{H}, A \in \mathfrak{A}^{\prime}, x_{0} \in X_{A}, f_{0} \in X^{*}$. If

$$
(-1)^{j-1} f_{0}\left(B_{k, j} x_{0}\right) \geqq 0 \quad\left(1 \leqq j \leqq m_{k}, 1 \leqq k<\infty\right)
$$

then $f_{0}\left(T(t) x_{0}\right) \geqq 0$ for all $t \geqq 0$.

Proof. From (5.3) and Lemma 2.7 we immediately have that $f_{0}\left(T_{p}(t) x_{0}\right) \geqq 0$. Now take $p \rightarrow \infty$.

TheOREM 5.3. Let $h \in \mathscr{H}^{\prime}, A \in \mathfrak{A}^{\prime}, x_{0} \in X_{A}, f_{0} \in X^{*}$. If (5.17) holds and, in addition,

$$
(-1)^{j-1} f_{0}\left(A B_{k, j} x_{0}\right) \geqq 0 \quad\left(1 \leqq j \leqq m_{k}, 1 \leqq k<\infty\right)
$$

then $f_{0}\left(T(t) x_{0}\right) \searrow$ if $t \nearrow$.

Note that $A B_{k, j} x_{0}$ is well defined for any $x_{0} \in X$.

Before proving this theorem, we state and prove the following theorem.

TheOREM 5.4. Let $h \in \mathscr{H}_{\infty}, A \in \mathfrak{U}^{\prime}, x_{0} \in X_{A}, f_{0} \in X^{*}$. Set

$$
\widetilde{B}_{k, j}^{n}=(-1)^{m_{k}-j-1} \sum_{i=0}^{j}\left(\begin{array}{c}
n \\
i
\end{array}\right) \mu_{k}^{n-i} B_{k, m_{k}-j-i} .
$$

If

$$
f_{0}\left(\widetilde{B}_{k, j}^{n} x_{0}\right) \geqq 0 \quad \text { for } 0 \leqq j \leqq m_{k}, 1 \leqq k<\infty, 0 \leqq n<\infty,
$$

then

$$
(-1)^{n} \frac{d^{n}}{d t^{n}} f_{0}\left(T(t) x_{0}\right) \geqq 0 \quad \text { for } 0 \leqq n<\infty, 0<t<\infty .
$$

Proof. Let $\Gamma$ be a circle about $\mu_{k}$ such that all the points $\mu_{j}$ with $j \neq k$ lie outside $\Gamma$. By the residue theorem,

$$
\begin{aligned}
\sum_{j=1}^{m_{k}} \frac{1}{(j-1) !} \frac{\partial^{j-1} S_{\mu_{k}}(t)}{\partial \mu^{j-1}} B_{k, j} & =\frac{1}{2 \pi i} \int_{\Gamma}(\lambda I-A)^{-1} S_{\lambda}(t) d \lambda \\
& =\frac{1}{2 \pi i} \int_{\Gamma} \lambda^{n}(\lambda I-A)^{-1}\left(\frac{S_{\lambda}(t)}{\lambda^{n}}\right) d \lambda \equiv J(t) .
\end{aligned}
$$

Set

$$
Q_{\lambda}^{n}(t)=(-1)^{n} \lambda^{-n} \frac{\partial^{n} S_{\lambda}(t)}{\partial t^{n}}
$$


and write

$$
\lambda^{n}=\sum_{i=0}^{n}\left(\begin{array}{c}
n \\
i
\end{array}\right) \mu_{k}^{n-i}\left(\lambda-\mu_{k}\right)^{i}
$$

From the residue theorem we then get

$$
\begin{aligned}
(-1)^{n} \frac{d^{n} J(t)}{d t^{n}}= & \frac{1}{2 \pi i} \int_{\Gamma}\left[\sum_{i=0}^{n}\left(\begin{array}{c}
n \\
i
\end{array}\right) \mu_{k}^{n-i}\left(\lambda-\mu_{k}\right)^{i}\right]\left[\sum_{j=1}^{m_{k}} \frac{B_{k, j}}{\left(\lambda-\mu_{k}\right)^{j}}\right] Q_{\lambda}^{n}(t) d \lambda \\
= & \sum_{j=1}^{m_{k}} B_{k, j} \sum_{i=0}^{j-1}\left(\begin{array}{c}
n \\
i
\end{array}\right) \mu_{k}^{n-i} \frac{1}{(j-i-1) !} \frac{\partial^{j-i-1} Q_{\mu_{k}}^{n}(t)}{\partial \mu^{j-i-1}} \\
= & \sum_{q=0}^{m_{k}-1}\left\{B_{k, m_{k}-q}\left(\begin{array}{l}
n \\
0
\end{array}\right) \mu_{k}^{n}+B_{k, m_{k}-q+1}\left(\begin{array}{c}
n \\
1
\end{array}\right) \mu_{k}^{n-1}+\cdots+B_{k, m_{k}}\left(\begin{array}{l}
n \\
q
\end{array}\right) \mu_{k}^{n-q}\right\} \\
& \times \frac{1}{\left(m_{k}-q-1\right) !} \frac{\partial^{m_{k}-q-1} Q_{\mu_{k}}^{n}(t)}{\partial \mu^{m_{k}-q-1}}
\end{aligned}
$$

where

$$
\left(\begin{array}{l}
n \\
i
\end{array}\right)=0 \quad \text { if } i>n
$$

Thus, by (5.19) the last sum is equal to

$$
\sum_{q=0}^{m_{k}-1}(-1)^{m_{k}-q-1} \frac{1}{\left(m_{k}-q-1\right) !} \frac{\partial^{m_{k}-q-1} Q_{\mu_{k}}^{n}(t)}{\partial \mu^{m_{k}-q-1}} \tilde{B}_{k, q}^{n} .
$$

Hence, recalling (5.22) and (5.3), we have

$$
(-1)^{n} \frac{d^{n} T_{p}(t) x_{0}}{d t^{n}}=\sum_{k=1}^{p} \sum_{q=0}^{m_{k}-1}(-1)^{m_{k}-q-1} \frac{1}{\left(m_{k}-q-1\right) !} \frac{\partial^{m_{k}-q-1} Q_{\mu_{k}}^{n}(t)}{\partial \mu^{m_{k}-q-1}} \widetilde{B}_{k, q}^{n} x_{0}
$$

Using Lemma 2.9 and the assumption (5.20), we conclude that

$$
(-1)^{n} \frac{d^{n} f_{0}\left(T_{p}(t) x_{0}\right)}{d t^{n}} \geqq 0 \quad \text { for } n=0,1,2, \ldots ; t>0 .
$$

Since $T_{p}(t) x_{0} \rightarrow T(t) x_{0}$ as $p \rightarrow \infty$, the assertion of the theorem follows from Lemma 2.3.

Using the notation $\Delta_{\eta}^{m}$ (following Lemma 2.3), we can state

COROLLARY. If (5.20) is assumed to hold only for $0 \leqq n \leqq n_{0}$, then

$$
(-1)^{n} \Delta_{\eta}^{n} f_{0}\left(T(t) x_{0}\right) \geqq 0 \quad \text { for } 0 \leqq n \leqq n_{0}, 0 \leqq t<t+\eta<\infty .
$$

Indeed, the proof of Theorem 5.4 shows that (5.24) holds with $T(t) x_{0}$ replaced by $T_{p}(t) x_{0}$. Since $T_{p}(t) x_{0} \rightarrow T(t) x_{0}$ as $p \rightarrow \infty$, (5.22) follows.

REMARK. Let the assumptions of Corollary 2 to Theorem 5.1 hold and let $h \in C^{n_{0}}[0, \infty)$. Then, by [6] and (5.14), $T(t) x_{0}$ has $n_{0}$ continuous derivatives in $[0, \infty)$. Hence, (5.24) implies that

$$
(-1)^{n}\left(\partial^{n} f_{0}\left(T(t) x_{0}\right) / \partial t^{n}\right) \geqq 0 \text { for } 0 \leqq n \leqq n_{0}, 0 \leqq t<\infty .
$$


Proof of Theorem 5.3. We shall use the formula

$$
T_{p}(t) x_{0}=\sum_{k=1}^{p} \sum_{q=0}^{m_{k}-1}(-1)^{m_{k}-q-1}\left[\frac{\partial^{m_{k}-q-1}}{\partial \mu^{m_{k}-q-1}}\left(\frac{S_{\mu}(t)}{\mu}\right)\right]_{\mu=\mu_{k}} \widetilde{B}_{k, q}^{1} x_{0}
$$

which one obtains by the same method that was used before to derive (5.23). Since

$$
\widetilde{B}_{k, q}^{1}=(-1)^{m_{k}-q-1}\left(\mu_{k} B_{k, m_{k}-q}+B_{k, m_{k}-q+1}\right) \quad\left(1 \leqq q \leqq m_{k}-1\right),
$$

(5.5) shows that the inequalities (5.18) imply the inequalities (5.20) for $n=1$. Hence, (5.26) gives

$$
f_{0}\left(T_{p}(t) x_{0}\right) \searrow \text { if } t \nearrow
$$

Since $T_{p}(t) x_{0} \rightarrow T(t) x_{0}$ as $p \rightarrow \infty$, the proof is complete.

REMARK. If $X$ is a finite-dimensional Banach space, then any linear operator $A$ whose eigenvalues are positive numbers is in $\mathfrak{A}^{\prime}$. Furthermore, the series in (5.2) now consists of a finite number of terms. Hence (5.7) holds. (5.8) is also valid; in fact, it easily follows using the residue theorem. Thus $X_{A}=X$.

6. Additional results. In the previous two sections we have derived theorems which involved the functions $S_{\lambda}(t)$ for $\lambda>0$. A crucial step in the derivation of these theorems was the behavior of the function $S_{\lambda}(t)$ for positive values of the parameter $\lambda$. Since analogous results on the behavior of $S_{\lambda}(t)$ for $\lambda$ complex are not available in the literature, we cannot extend, at present, the results of $\$ \$ 4,5$ to operators $A$ with $\sigma(A)$ which is not contained in the real interval $0<\lambda<\infty$.

However, for some special functions $h(t)$, the behavior of $S_{\lambda}(t)$, for complex $\lambda$, is known with sufficient precision. We give here one example where $h(t)=t^{-\alpha}$ for some $0<\alpha<1$. Then $S_{\lambda}(t)=E_{\beta}\left(-\gamma \lambda t^{\beta}\right)$ where $\beta=1-\alpha, \gamma=\Gamma(\beta)$ and where $E_{\beta}(z)$ is the Mittag-Leffler function

$$
E_{\beta}(z)=\sum_{n=0}^{\infty} \frac{z^{n}}{\Gamma(n \beta+1)}
$$

From a well-known asymptotic formula for $E_{\beta}(z)$ (see [1, p. 207]) we find that

$$
\left|S_{\lambda}(t)-\frac{\gamma_{0}}{\lambda t^{\beta}}\right| \leqq \frac{c}{|\lambda|^{2} t^{2 \beta}} \text { if }\left|\arg \lambda t^{\beta}\right|<\frac{1+\alpha}{2} \pi
$$

provided $\left|\lambda t^{\beta}\right| \geqq c_{0}>0$; here $\gamma_{0}=(\Gamma(\alpha) \Gamma(1-\alpha))^{-1}$.

Let us assume that the resolvent set $\rho(A)$ of $A$ contains the sector $|\arg \lambda|>$ $(1+\alpha) \pi / 2$ and that $\left\|(\lambda I-A)^{-1}\right\| \leqq c /(1+|\lambda|)$ for $\lambda$ in this sector. We define $S(t)$ by (3.23) and choose $C$ in $\rho(A)$ such that (6.1) holds for $\lambda \in C$. It follows that $S(t)$ is a bounded operator for each $t>0$. Furthermore, it varies continuously in $t$. One can also continue $S(t)$ analytically into a sector $|\arg \lambda|<\delta$ for some $\delta>0$.

Since $h(0)=\infty$, the results of [6] do not cover the present case of $h(t)=t^{-\alpha}$. There arises the question in what sense is $S(t)$ a fundamental solution. 
So far we have only considered solutions of equations of the form (1.1) where $x_{0}$ is independent of $t$. But some of the results extend without difficulty to the equations

$$
x(t)=k(t) x_{0}-\int_{0}^{t} h(t-\tau) A x(\tau) d \tau
$$

where $k(t)$ is a scalar function.

The solution is given by (see [6]):

$$
x(t)=k(0) S(t) x_{0}+\int_{0}^{t} k(\tau) S(t-\tau) x_{0} d \tau
$$

where $k(\tau)=d k(\tau) / d \tau$. Hence, if $X$ is a Hilbert space,

$$
\left(x(t), x_{0}\right)=k(0)\left(S(t) x_{0}, x_{0}\right)+\int_{0}^{t} \dot{k}(\tau)\left(S(t-\tau) x_{0}, x_{0}\right) d \tau .
$$

This relation combined with the results of $\$ \$ 4,5$ yields monotonicity properties for $\left(x(t), x_{0}\right)$. For example, if $k(0) \geqq 0, \dot{k}(\tau) \geqq 0$, then $\left(x(t), x_{0}\right) \geqq 0$.

Applications. If $h \in \mathscr{H}_{\infty}$ then we have proved several theorems to the effect that $\left(S(t) x_{0}, x_{0}\right)$ is completely monotonic in $t$. Since $\left(S(0) x_{0}, x_{0}\right)=\left(x_{0}, x_{0}\right) \neq 0$, we conclude that $\left(S(t) x_{0}, x_{0}\right)>0$ for all $t>0$. In particular, $S(t) x_{0} \neq 0$ for all $t>0$. Thus the solutions of (1.1) have the "weak backward uniqueness" property as defined in [5]. This fact is important in the study of optimal-control for trajectories $x(t)$ given by

$$
x(t)=u(t)+\int_{0}^{t} h(t-\tau) A x(\tau) d \tau
$$

where $u(t)$ is the control function. It enables us to prove uniqueness of timeoptimal controls (see [3], [5, p. 42]).

If $A$ is selfadjoint and if $h \in \mathscr{H}^{\prime}$ and $h$ is strictly decreasing, then we can again assert that $\left(S(t) x_{0}, x_{0}\right)>0$ for all $x_{0} \neq 0, t>0$. Indeed, otherwise we get, from (4.1), $S_{\mu}\left(t_{0}\right)=0$ for some $\mu>0, t_{0}>0$. But then, by Lemma $2.5, S_{\mu}(t)=0$ if $t>t_{0}$. Using (2.4) we then see that $S_{\mu}(t)<S_{\mu}\left(t_{0}\right)$ if $t>t_{0}$; a contradiction.

If $h \in \mathscr{H}^{\prime}$, then we have proved several theorems to the effect that $\left(S(t) x_{0}, x_{0}\right) \searrow$ if $t \nearrow$. This can be used to answer some questions of controllability; for instance, to show that a point $x_{0}$ can be "steered," by a suitable control, to any given neighborhood of 0 (cf. [3]).

\section{REFERENCES}

1. A. Erdélyi et al., "Higher transcendental functions," in Bateman manuscript project, Vol. III, McGraw-Hill, New York, 1955.

2. A. Friedman, On integral equations of Volterra type, J. Analyse Math. 11 (1963), 381-413.

3. - Optimal control for hereditary processes, Arch. Rational Mech. Anal. 15 (1964), 396-416. 
4. A. Friedman, Periodic behavior of solutions of Volterra integral equations, J. Analyse Math. 15 (1965), 287-303.

5. —- Optimal control in Banach spaces, J. Math. Anal. Appl. 19 (1967), 35-55.

6. A. Friedman and M. Shinbrot, Volterra integral equations in Banach space, Trans. Amer. Math. Soc. 126 (1967), 131-179.

7. T. Kato, Fractional powers of dissipative operators, J. Math. Soc. Japan 13 (1961), 246-274.

8. R. K. Miller, On Volterra integral equations with non-negative integrable resolvents, J. Math. Anal. Appl. 22 (1968), 319-340.

9. D. V. Widder, The Laplace transform, Princeton Univ. Press, Princeton, N. J., 1946.

NORTHWESTERN UNIVERSITY,

EVANSTON, ILLINOIS 